\title{
Coulisses
}

Revue de théâtre

40 | Hiver 2010

Jeux de rappels chez Marivaux

\section{Trois pièces classiques}

Fantasio, Le Bourgeois Gentilhomme, La seconde surprise de l'amour

\section{David Ball}

\section{(2) OpenEdition}

\section{Journals}

Édition électronique

URL : https://journals.openedition.org/coulisses/635

DOI : $10.4000 /$ coulisses.635

ISSN : 2546-9460

Éditeur

Presses universitaires de Franche-Comté

\section{Édition imprimée}

Date de publication : 1 janvier 2010

Pagination : 13-18

ISBN : 978-2-84867-283-0

ISSN : 1150-594X

Référence électronique

David Ball, «Trois pièces classiques », Coulisses [En ligne], 40 | Hiver 2010, mis en ligne le 30

novembre 2016, consulté le 29 décembre 2022. URL : http://journals.openedition.org/coulisses/635 ;

DOI : https://doi.org/10.4000/coulisses.635

Ce document a été généré automatiquement le 29 décembre 2022.

Tous droits réservés 


\section{Trois pièces classiques}

Fantasio, Le Bourgeois Gentilhomme, La seconde surprise de l'amour

\section{David Ball}

\section{RÉFÉRENCE}

Fantasio d'Alfred de Musset au Nouveau Théâtre de Besançon, mise en scène de Julia

Vidit, avril 2009

Le Bourgeois Gentilhomme de Molière et Lully au Théâtre musical de Besançon

Direction artistique et musicale : Vincent Dumestre, mise en scène : Benjamin Lazar.

Chorégraphie : Cécile Roussat, 2009

La seconde surprise de l'amour de Marivaux à la Commanderie de Dole

Mise en scène : Luc Bondy, interprétation : Pascal Bongard, Audrey Bonnet, Roger

Jendly, Roch Leibovici, Micha Lescot, Marie Vialle, juin 2009

\section{Fantasio d'Alfred de Musset}

1 Calquée sur les deux actes de la pièce, la mise en scène est en au moins deux parties distinctes. La première est sombre et désespérée : Fantasio et ses amis assis sur un cercueil devant un haut mur qui les confine et les domine. Le cercueil est celui de SaintJean, le bouffon du roi qui vient de mourir et que Fantasio (joué par Jean-Baptiste Verquin) remplacera. On fait sortir le cercueil vers la fin du premier acte, et quand Fantasio entre en scène de nouveau, déguisé en bouffon de manière fantasque et grotesque, son jeu, pour souligner peut-être l'effet de contraste, s'apaise. Là où désoeuvré il criait et gesticulait, maintenant que c'est son déguisement qui crie, sa voix s'adoucit. Il était temps! On s'impatientait de voir et d'entendre s'exprimer le côté poétique, shakespearien, de la pièce.

2 Fantasio en bouffon est un avatar du clown shakespearien, dans le style des rôles que Shakespeare avait créés dès 1599 pour l'acteur Robert Armin qui, de toute évidence, partageait avec son auteur le goût du calembour, et de plus savait chanter. Le rôle de 
Feste dans La Nuit des rois est probablement l'exemple le plus connu et le plus apprécié de ce style de clown plutôt philosophique, où l'humour s'exprime bien plus par la parole que par le geste ou par l'acrobatie.

3 Le nœud de l'intrigue de Fantasio, par contre, vient de Marivaux. C'est l'échange de rôles entre le duc et son aide de camp, pour que le maitre puisse juger le caractère de sa promise avant de s'engager définitivement, qui rappelle le double échange du Jeu de l'amour et du hasard. Malheureusement, le jeu du roi, le père de la promise, et celui du duc dépassent par leurs exagérations toutes bornes imaginables, du bon sens surtout et du respect du texte.

4 Tout cela se passe, pourtant, dans un joli décor. Quand le mur tombe, c'est un jardin qui nous est révélé, jardin qui est aussi un dédale, composé d'écrans ou de paravents transparents et surmontés de fleurs (décors signés Thibaut Fack). Quelques costumes princiers aussi sont magnifiques de couleur et de panache (création costumes : Valérie Ranchoux).

5 Mais à la fin, Fantasio, dans sa cellule, retrouve l'ambiance sombre du début de la pièce. Pourquoi se trouve-t-il en prison? Il a pêché au bout d'une ficelle la perruque du duc! Les fiançailles sont rompues en conséquence de cette instance de lèse-majesté, et la guerre va se déclarer, une menace qui n'est sans doute pas à prendre trop au sérieux. Fantasio révèle à la princesse qu'il a adopté le rôle de bouffon uniquement pour échapper à ses créanciers, mais ils se mettent d'accord pour qu'il revienne au jardin déguisé en bouffon pour amuser la princesse chaque fois qu'il lui plaira. La mise en scène va, pourtant, plus loin que cela, pour tomber dans un romantisme, ou plutôt dans un sentimentalisme, qui n'est pas celui de Musset. La petite promesse de complicité et de bonheur de sa fin à lui est ici gonflée et déformée par le désespoir de la princesse à voir partir un Fantasio dont elle est visiblement tombée amoureuse.

6 Il faut, pourtant, saluer l'énergie et l'engagement des acteurs, qui assurent tous, à l'exception de Jean-Baptiste Verquin (Fantasio), plus d'un rôle. C'est du Musset en dessin animé peut-être mais la poésie est toujours là, même submergée, et le côté dessin animé marche à sa manière, surtout, sans doute, aux yeux d'un jeune public.

\section{Le Bourgeois Gentilhomme de Molière et Lully}

7 Belle leçon d'histoire, tout de même, cette recréation fidèle des premières représentations de la pièce, même si ses aspects pédants risquent d'être un brin infidèles à l'esprit de notre auteur. Tout est de l'époque : décor, costumes, éclairages, même la prononciation se veut authentique, avec les $s$ et les $t$ terminaux bien audibles, les voyelles nasales prononcées comme à Marseille et les $r$ roulés comme en Bourgogne, ce qui ajoute une couche comique supplémentaire à tous les personnages mais qui tend à effacer les contrastes entre eux. Le décor spacieux et neutre est celui d'un théâtre néoclassique, ses murs dorés et décorés pour l'occasion. On saluera notamment l'originalité du décor mobile du souper avec la "marquise», où les cuisiniers transformés en derviches-tourneurs et les tables, elles aussi tournantes, créent un tableau à mi-chemin entre danse, féérie et fête galante. Les costumes, toujours somptueux mais respectant soigneusement les différences sociales, sont de tous les jours pour les parties parlées et de fantaisie pour les parties chantées et dansées, et 
l'éclairage imite la lumière douce et atténuée des chandelles. (Décor: Adeline Caron, costumes : Alain Blanchot, lumières : Christophe Naillet.)

8 Le spectacle est beau, la musique de Lully belle, copieuse et insistante mais belle (tout ce qui est baroque est beau aujourd'hui, n'est-ce pas?), et les danses mettent bien en valeur les talents des exécutants. Si les danses de cour sont un peu répétitives, le ballet d'ouverture à l'arrivée des turcs est magistral, haut en couleurs et en mouvements d'ensemble.

9 Force est de constater, pourtant, que la comédie-ballet est une forme hybride imposée par le roi et qui nuit ici à la comédie, à la pièce de Molière, qui est en elle-même une vraie comédie de mœurs : elle parle des changements sociaux importants de l'époque et satirise du point de vue typiquement conservateur de la comédie les prétentions d'une bourgeoisie montante. Prétentions qui étaient ridicules uniquement dans la mesure où elles imitaient les manières d'une aristocratie vieillissante, la bourgeoisie étant une classe nouvelle, déjà puissante, qui n'avait pas besoin de singer celle qu'elle allait remplacer.

Il y a une légende que Shakespeare écrivit Les Joyeuses Commères de Windsor à la demande de la reine Elisabeth, qui voulut revoir Falstaff, mais en amoureux. La pièce, écrite en deux semaines, toujours selon la légende, n'est pas parmi les plus grandes de son auteur mais c'est du Shakespeare, c'est une vraie pièce de théâtre, elle n'est pas affublée d'un ballet qui n'a rien à voir avec l'intrigue.

11 Molière se plia aux exigences de Louis XIV et collabora aussi intelligemment et loyalement que possible avec Lully mais le résultat déforme et étire sa comédie qui, sans musique ni danse, aurait pu très bien marcher toute seule. Nous pouvons dire à peu près la même chose du Malade imaginaire, comédie-ballet écrite cette fois-ci en collaboration avec Charpentier. Une satire percutante à propos de la bêtise et de l'incompétence meurtrières des médecins ne se prête pas forcément à chanter et à danser, surtout que le sérieux de son propos a été accentué accessoirement par la maladie de Molière lui-même, maladie mortelle qui allait le frapper en pleine représentation de cette même pièce.

12 Toutes deux, certes, sont des comédies qui tirent sur la farce, mais tout comme elles n'ont pas besoin d'être interrompues par de la musique, elles n'ont pas besoin non plus d'un jeu débordant de caricature. Un tel jeu, comme c'est malheureusement le cas dans cette mise en scène, a parfois tendance, lui aussi, à faire perdre au texte de Molière sa pertinence. Le bourgeois gentilhomme lui-même (joué avec talent et énergie par Olivier Martin Salvan) s'impose tout de même, mais le personnage de son épouse, joué ici par un homme (Nicolas Vial) comme une « dame » de la pantomime anglaise, est ainsi privé de tout le bon sens qui est censé faire contraste aux extravagances de son mari. Cependant, un tel choix a le mérite de souligner justement le caractère farcesque du couple Jourdain, ainsi bien sûr que de rappeler combien Mme Jourdain est une maîtresse femme, comme tant d'autres chez Molière, tout en soulignant par contraste la fraîcheur d'une Dorimène...

13 Et puis le tout, musique, danse et clowneries comprises, est d'une longueur éprouvante, particulièrement pour ce qui concerne le divertissement auquel assistent les personnages eux-mêmes. Ce fut après minuit que le public bisontin put enfin quitter un théâtre chaud comme un bain turc! 
Mais l'expérience, même si elle n'est pas tout à fait concluante, reste intéressante à bien des égards, superbe esthétiquement et, compte tenu du talent et de l'implication de tous les participants, admirable. Nous leur savons gré à tous de nous avoir offert cette possibilité de voir la comédie-ballet dans tout son faste. Notre dernier mot doit être, en toute honnêteté, en toute modestie, celui de la reconnaissance.

\section{La seconde surprise de l'amour de Marivaux}

On parlerait aujourd'hui de l'inconscient freudien ou du désir lacanien toujours reporté là où au dix-huitième siècle on parlait du cœur et de la raison en conflit inévitable. Et un aspect du succès de Marivaux dans cette pièce est de faire parler à tous ses personnages principaux et le cœur et la raison. Lisette, tout comme la Colombine de la première Surprise de l'amour, le fait en toute connaissance de cause pour faire aboutir son mariage avec Lubin comme conséquence bien naturelle du mariage de sa maitresse avec le Chevalier, le maître de Lubin. La Marquise et le Chevalier croient toujours parler raison mais c'est en effet le cœur qui domine, un cœur d'abord noyé dans le chagrin d'un amour perdu et ensuite enflammé par la jalousie ou l'amour-propre, jusqu'à la dernière scène, le moment de la surprise, quand l'éclaircissement si attendu enfin se fait. Incertitude ravissante, la possibilité d'une hypocrisie calculée de la part de ces deux amants! De toute manière, les acteurs savent bien mieux que nous, les spectateurs, vers quel dénouement on est en train de se diriger, et l'équilibre qu'ils ont à maintenir est de rester tout entiers dans l'inconscience ou la mauvaise foi des personnages et en même temps de provoquer la complicité d'un public ayant déjà suffisamment compris. Suffisamment mais pas trop. Si, lors de la dernière scène, nous avons l'impression que la brouille, vraie ou fausse, entre la Marquise et le Chevalier a été trop vivement exprimée pour être rattrapée, la surprise sera là pour nous aussi, et la satisfaction avec, de voir tout s'éclaircir si rapidement.

Voilà pour le Marivaux du dix-huitième siècle, celui du texte. Passons maintenant à celui du vingt-et-unième, de la mise en scène de Luc Bondy. Les deux amants sont ici deux têtes de linotte, des caricatures de jeunes d'aujourd'hui, irréfléchis, irrespectueux, boudeurs, égoïstes. Lisette et Lubin sont encore plus voyous. La scène où ils chassent Hortensius et jettent ses livres par terre rappelle des passages du film de Kubrick, Orange mécanique, dans la brutalité de leur insouciance et de leur désinvolture. Mais faire de La Seconde Surprise de l'amour, même si elle est un peu moins gaie, un peu moins gentille, que la première, une pièce anti-jeunes est un peu trop dans l'air du temps, je trouve, surtout que l'élégance du décor, par contraste avec les agissements des personnages, semble enfoncer le clou de la critique.

Décor et costumes (signés Karl-Ernst Herrmann et Moidele Bickel respectivement) forment un ensemble, en trois couleurs : noir, blanc et gris, à part le pantalon jaune du Chevalier. Le décor se présente comme un tableau, abstrait, minimaliste, un peu surréaliste: au milieu du plateau, une longue estrade avec à chaque extrémité une cabane de plage noire, côté jardin celle de la Marquise, côté cour celle du Chevalier. Et vers la fin de la pièce, les deux cabanes sont tirées l'une vers l'autre, l'une dans l'autre : symbole!

18 Si les acteurs principaux ont tendance à parler trop vite pour le grand espace de la Commanderie, plus adapté aux spectacles de variétés, le rythme de la mise en scène est souvent trop lent, et nous avons du mal à nous intéresser jusqu'au bout à ces 
personnages à l'air si bête, si frivole. L'intelligence de l'auteur est toujours là, bien sûr, mais si en plus on a du mal à entendre les répliques...?

Des trouvailles malgré tout, c'est indéniable. Le jeu sur l'arrière-plan, qui multiplie l'unité de temps et de lieu, clin d'œil au cinéma ; la bicyclette de Lubin, à l'unisson avec le mouvement général de la pièce, rapide et tourbillonnant; le noir, qui rappelle la rapidité des vingt-quatre heures passées à tomber amoureux, et qui baigne tout le dernier acte, suggérant du même coup toute la cruauté de cette pièce.

20 Car, dans sa concentration sur l'essentiel, quel dramaturge impitoyable tout de même, ce Marivaux! Le mari décédé et la bien aimée réfugiée au couvent, dont l'après-vie aurait pu être encombrante : oubliés! Le pauvre Comte, qui n'a pas démérité en suscitant la jalousie du Chevalier : congédié ! Hortensius le pédant, de même. Même en tant que personnage comique, il n'a pas le droit d'ennuyer une grande dame, en lui enseignant une philosophie censée être remède contre les passions. Sa sincérité, pourtant, son désir de plaire, très évidents dans cette mise en scène, ne sont pas récompensés comme il se doit. Il y a là de la dureté : dans le texte de Marivaux, le pouvoir d'une classe sociale dominante, et dans la mise en scène de Luc Bondy, une jeunesse insolente. La grâce, le raffinement, la politesse de cette classe dominante, cependant, même si nous savons aujourd'hui que ces qualités étaient pour la plupart superficielles et mal réparties dans un siècle qui allait se terminer par la Révolution, il nous faut néanmoins trouver les moyens de leur faire justice, non plus en tant que privilèges réservés à une élite aristocratique mais accessibles en principe à tous, comme la promesse d'un monde enfin civilisé.

\section{INDEX}

oeuvrecitee Fantasio, Bourgeois Gentilhomme (Le), Seconde surprise de l'amour (La) 